\title{
EFFECT OF PREPARATION DESIGN ON FRACTURE RESISTANCE AND FAILURE MODE OF ROOT CANAL TREATED MAXILLARY PREMOLARS RESTORED WITH ONLAY RESTORATIONS
}

\author{
Walid Al-Zordk*
}

\begin{abstract}
Purpose: Cusp fractures are among the major reasons for the extractions of root-treated teeth. With the intention of increasing information about the mechanical behavior of minimally invasive cusp coverage restorations, the aim of current study was to evaluate the fracture resistance and failure modes of endodontically treated maxillary premolars restored with onlays employing various cusp reduction designs (anatomic, horizontal and beveled) to identify the optimum design and to assess whether these designs influence the restorable fracture rate.
\end{abstract}

Materials and methods: Fifty defect-free maxillary premolar teeth were divided into 5 groups: onlay with anatomic occlusal reduction design (OA), onlay with horizontal occlusal reduction design $(\mathrm{OH})$, onlay with beveled occlusal reduction design $(\mathrm{OB})$, full-coverage crown $(\mathrm{CR})$ and non-restored sound teeth (NR). In $\mathrm{OA}, \mathrm{OH}, \mathrm{OB}$ and $\mathrm{CR}$ groups, the access cavities were sealed using bonded composite after endodontic treatment. For OA, OH and $\mathrm{OB}$ groups, cusps were prepared with anatomic, horizontal and beveled designs. Except for NR group, all teeth were restored with resin-infiltrated ceramic restorations. After thermal aging for 10000 cycles between $5^{\circ} \mathrm{C}$ and $50^{\circ} \mathrm{C}$, all specimens were submitted to compressive axial load at a crosshead speed of 1 $\mathrm{mm} \mathrm{min}^{-1}$ till failure. The data were analyzed using ANOVA test.

Results: The highest load to fracture values $(\mathrm{N})$ was recorded in control non-restored group $(1657 \pm 167.7 \mathrm{~N})$, followed by $\mathrm{CR}, \mathrm{OA}, \mathrm{OB}$ and $\mathrm{OH}$ groups where the mean values were $887.5 \pm 40.3$, $789.4 \pm 54.8,722.2 \pm 46.2$ and $634.8 \pm 74.2 \mathrm{~N}$ respectively. Significant differences were found among fracture values of test groups $(p<.05)$. The intact teeth predominantly fractured with favorable fracture patterns followed by $\mathrm{CR}$ group; while both $\mathrm{OB}$ and $\mathrm{OH}$ groups showed the heights nonfavorable pattern of fracture. Group OA represented moderate percentage of favorable fracture.

Conclusions: Root treated teeth restored with anatomic cusp reduction design displayed greater fracture resistance and greater rates of restorable fractures than root treated teeth submitted to horizontal and beveled reduction deigns.

KEY WORDS: Onlay, endodontically treated, ceramics, cusp coverage.

\footnotetext{
* Assistant professor, Department of Fixed Prosthodontics, Mansoura University, Mansoura, Dkahlia, Egypt
} 


\section{INTRODUCTION}

The restoration of root treated tooth is still a much-debated topic. The endodontic treatment results in decline in the strength of the remaining tooth structure because of multitude of factors such as alterations in tooth architecture, alterations in the properties of dentin and alterations in proprioception. The alterations in tooth architecture are assigned to removal of tooth structure during endodontic access preparation with subsequent weakening of the tooth and more prone to fracture. ${ }^{1,2}$ The endodontic treatment is associated with reduction of proprioceptive sensation in non-vital tooth with higher pain threshold, as a consequence of pulpal nerves being concerned in detecting masticatory load, with subsequent elevated loading of nonvital tooth. ${ }^{3}$ Collagen depletion have an impact on the elasticity of the dentin and raises the change of fracture under occlusal forces. During endodontic protocol, certain steps have a negative influence on collagen involving the use of sodium hypochlorite, ethylenediamine tetra acetic acid and calcium hydroxide dressing., 4

For restoration of endodontically treated teeth, the ideal definitive restoration is dependent on the remaining tooth structure as the strength of the tooth depends on the amount of remaining hard dental tissue. ${ }^{6}$ Intra-coronal restoration is only proposed if mesial and distal marginal ridges are intact. ${ }^{7} \mathrm{~A}$ popular approach for restoration of endodontically treated teeth has been to construct an intra-radicular post and core build up to enhance the retention of a definitive restoration. With this protocol, either prefabricated or custom-made intra-radicular posts can be utilized. The principle function of a post is to provide intra-radicular retention of the core/ crown restoration and to distribute functional loads to a larger area of the remaining tooth structure. ${ }^{8}$ However, post do not strengthen the endodontically treated teeth. ${ }^{2,9}$ Plentiful researches have pointed out that placement of an intra-radicular post will aid in the retention of the definitive restoration but may have a weakening influence on the tooth.,.$^{\mathbf{8 1 0}}$ Furthermore, the implementation of post and core approach is complicated by the essential need to provide an appropriate ferrule effect, which is essential to lessen the danger of failure with root fracture. ${ }^{11}$ The ferrule effect is a key factor in the failure threshold of post-treated teeth, but the same ferrule preparation also causes loss of important remaining hard dental tissues. ${ }^{12}$ Stankiewicz et al ${ }^{13}$ demonstrated that a ferrule is desired, but should not be provided at the expense of the remaining tooth/root structure. Failure related to post and core possible attributed to various biomechanical behaviors of tooth structure as a consequence to intra-oral repeated stresses. ${ }^{14}$ The failure may be repairable or non-repairable that demands tooth removal and later prosthetic substitution. ${ }^{15}$

With modern developments in adhesive methods and restorative materials, existence of macroretentive features is no longer a pre-requisite for adhesively bonded restoration if there are enough tooth structure for bonding. ${ }^{16,17}$ A change in treatment protocols to more conservative decisions has been advocated, and the requirement for intraradicular post and core has become less evident. ${ }^{18}$ Depending on the available tooth structure, inlay, onlay and endo-crown restorations have been presented as an alternate option for restoration of root treated posterior teeth. ${ }^{19,20}$ Based on the adhesive techniques, minimally invasive preparation designs which preserve as much tooth structure as possible are believed to be the gold standard in restoring a tooth. ${ }^{21,22}$ Onlay restoration is outlined to cover part or all of the occlusal aspect of the tooth and some authors apply the term overlays to describe full-occlusal coverage restorations. Traditional metal onlay restoration was designed to include an intra-coronal inlay component to provide adequate retention when conventional cementation was utilized. These days, onlay restorations are constructed from an assortment of 
restorative materials such as ceramic and composite materials. ${ }^{2324}$ An onlay restoration with cuspal coverage may efficiently restore an endodontically treated posterior tooth. Proximal box can enhance the retention and resistance of an only restoration but it causes more destruction and should only be implemented when previous restoration is existing. ${ }^{25}$

Cusp fractures are among the major reasons for the extractions of endodontically treated teeth. ${ }^{26}$ Unrestorable tooth fracture is noticed more frequently in endodontically treated tooth with massive intra-coronal restoration..$^{22,27,28}$ Studies has pointed out variety of factors, which affect the direction and location of root fracture, such as the restorative procedure, the preparation design and the remaining tooth structure. ${ }^{29,30,31}$ With the purpose of rising the amount of information concerning the mechanical impact of minimally invasive cusp coverage restorations, the present study evaluated the fracture resistance and failure modes of endodontically treated maxillary premolars restored with onlay employing various cusp reduction designs (anatomic, horizontal and beveled) to identify the optimum design and to assess whether these designs influence the ability to restore fractures. The null hypothesis examined was that cusp preparation design would have no effect on the fracture resistance and fracture mode of onlay restorations of endodontically treated maxillary premolars.

\section{MATERIALS AND METHODS}

\section{Teeth collection and grouping:}

Fifty human maxillary premolar teeth, extracted for periodontal reasons, with approximately similar coronal dimensions (bucco-lingually, mesiodistally and occluso-cervically measured from cemento-enamel junction), were sampled in the current investigation. The teeth were selected to be of similar roots with fully developed apices, noncarious, free form cracks. The selected teeth were debrided and cleaned of calculous and soft tissue. All the teeth had been collected in the Oral and Maxillofacial Department, Faculty of Dentistry, Mansoura University. The selected teeth were stored in $0.1 \%$ thymol solution at room temperature for one day, then stored in distilled water for no longer than 3 months. All selected teeth were randomly allocated into four equal groups of 10 teeth based on the preparation design: onlay with anatomic occlusal reduction design (OA), onlay with horizontal occlusal reduction design $(\mathrm{OH})$, onlay with beveled occlusal reduction design (OB), full-coverage crown (CR) and non-restored sound teeth (NR).

\section{Endodontic protocol:}

Teeth in $\mathrm{OA}, \mathrm{OH}, \mathrm{OB}$ and $\mathrm{CR}$ groups were endodontically treated following standardized procedures. Standard straight access was performed utilizing round bur and rounded end tapered burs. The canal preparation was done with a machine-driven rotary (ProTaper Dentsply Maillefer, Swtzerland). After each file, the canal was irrigated with $2.5 \%$ sodium hypochlorite solution for 10 seconds. The canal was dried with paper points (Aborbent Paper point, Meta Biomed Co Ltd, Korea) and obturated with gutta percha (Gutta Percha Points, Meta Biomed Co Ltd, Korea) and root canal sealer (Adseal Root Canal Sealer, Meta Biomed Co Ltd, Korea) according to manufacturer's instructions. Then, the superior aspect of gutta percha was removed to $2 \mathrm{~mm}$ bellow the cemento-enamel junction. The access cavity was fully restored with composite resin (Nexocomp, Meta Biomed Co Ltd, Korea). Then, all teeth were individually fixed in acrylic resin (Acrostone, Acrostone Dental Manufacture, Egypt) to a level $2 \mathrm{~mm}$ apical to the cemento-enamel junction. A silicon index (Ghenesyl, Lascod, Italy) was fabricated for each tooth and was sectioned in bucoo-lingual direction and used to control tooth structure removal and check restoration contour. 


\section{Tooth preparation:}

The MOD cavity was prepared without proximal steps. The preparation had an isthmus width of half the bucc-lingual distance. The pulpal floor was prepared to be at right angles with the long axis of the tooth and placed $1.5 \mathrm{~mm}$ above the cementoenamel junction. The preparation was performed with large-grit diamonds and finishing was performed with finer diamonds (Inlay Preparation Set, Komet). The cavity dimensions were measured with digital caliper $(150 \mathrm{~mm} / 6 \mathrm{in}$, American Spares Industriz). For teeth in OA group, the cusps were reduced parallel to the cusp inclines. For teeth in $\mathrm{OH}$ group, the cusps were reduced in parallel with the occlusal plane with no bevel. For teeth in $\mathrm{OB}$ group, the cusps were prepared in parallel with the occlusal plane, then bevel reductions were accomplished in a reverse angle to the natural cusp ridge. ${ }^{32,33}$ For teeth in group $\mathrm{CR}$, the preparation was performed to receive a full coverage crown: 1-mm circumferential chamfer margin placed $0.5 \mathrm{~mm}$ above cemento-enamel junction and 1.5 $\mathrm{mm}$ anatomical occlusal reduction. The sectioned putty index was used to assess the preparation. All preparation was performed by single operator with the handpiece stabilized in a parallelometer.

\section{Restoration fabrication and luting procedures}

CAD-CAM ceramic restorations were fabricated from resin-infiltrated ceramic (vita Enamic, Vita Zahnfabrik, Germany) using Ceramill motion 2 CAD-CAM system (Amann Girrbach $\mathrm{GmbH}$ ). All restorations were fabricated to have the same occluso-gingival height as well as having similar occlusal morphology. After each restoration was checked for accuracy of fit, intaglio surface of restorations was etched with $5 \%$ hydrofluoric acid gel (IPS Ceramic Etching-gel, Ivoclar Vivadent) for 60 seconds according to manufacturer's instructions, then rinsed with for 60 seconds and dried with moist-free air. Then, silane coupling agent (Porcelain Silane, Ultradent Products) was applied and allowed to dry for 60 seconds. The enamel of the prepared tooth surfaces was selectively etched with 37\% phosphoric acid gel (Meta Etchant, Meta Biomed) for 30 seconds, then rinsed and dried. All restorations were cemented with self-adhesive resin cement (Panavia SA Cement Plus, Kurary, Japan). The resin cement was applied to the intaglio surface of the restorations which was seated on its corresponding prepared tooth by static finger pressure then under constant axial load of $1 \mathrm{~kg}$. After brief light curing, excess cement was removed. Then, light curing was done at each surface for 20 seconds. Margins of the restoration were finished with fine finishing diamond. Then, the specimens were stored in distilled water for one week at room temperature. Specimens were subjected to 10000 thermal cycles between 5 and $55^{\circ} \mathrm{C}$ with a dual time of 30 seconds at each temperature in the thermal cycling machine (Thermocycler, SD Mechatronik, Germany).

\section{Fracture resistance test}

Each specimen was subjected to fracture test using universal testing machine (Instron, Instron Corp., Canton, MA, USA). Force was applied through a 5-mm stainless steel ball to the center of the occlusal surface and contacting the buccal and lingual triangular ridge representing the antagonistic tooth. ${ }^{34} \mathrm{~A}$ tin foil was affixed the ball to distribute the force regularly. Load was applied at a cross speed of $1 \mathrm{~mm} \mathrm{~min}{ }^{-1}$ till failure. The fracture load to failure was recorded in newtons $(\mathrm{N})$. For each specimen, pattern of failure was inspected and classified based on the location of fracture. Favorable failure was defined as repairable failure above the level of osseous simulation and included adhesive failure. Whereas, unfavorable failure was recognized as non-repairable and catastrophic failure below the level of osseous simulation.

\section{Statistical interpretations}

Statistical analysis was done using statistical software (SPSS Statistics for Windows v17.0 SPSS Inc). 


\section{RESULTS}

Means and standard deviations of load to fracture values are presented in Table 1. Normal and relative (marginal) distributions of data was tested by using Kolmogorov-Smirnov and Levene normality tests that revealed normal data distribution. Therefore, parametric one-way ANOVA test was conducted to assess the difference in load to fracture values among experimental group (Table 2). The highest overall load to fracture values $(\mathrm{N})$ was recorded in control non-restored group $(1657 \pm 167.7 \mathrm{~N})$, followed by $\mathrm{CR}, \mathrm{OA}, \mathrm{OB}$ and $\mathrm{OH}$ groups where the mean values were $(887.5 \pm 40.3,789.4 \pm 54.8$, $722.2 \pm 46.2$ and $634.8 \pm 74.2 \mathrm{~N}$ ) respectively. Significant differences were found among load to fracture values of experimental groups under study with $\mathrm{CR}$ group showed the height values $(P<.05)$.

Fracture pattern percentages of the experimental groups are represented in Table 3 and in figures 1 \& 2. The intact teeth predominantly fractured with favorable fracture patterns followed by $\mathrm{CR}$ group; while both $\mathrm{OB}$ and $\mathrm{OH}$ groups showed the heights non-favorable pattern of fracture. Group OA represented moderate percentage of favorable fracture.

TABLE (1) Mean load to fracture and standard deviation (in Neoton) for each group.

\begin{tabular}{|c|c|c|c|}
\hline Groups & Mean & Std. Deviation & $\mathrm{N}$ \\
\hline OA & 789.4 & 54.8 & 10 \\
\hline OH & 634.8 & 74.2 & 10 \\
\hline OB & 722.2 & 46.2 & 10 \\
\hline CR & 887.5 & 40.3 & 10 \\
\hline NR & 1657 & 167.7 & 10 \\
\hline
\end{tabular}

TABLE (2) one-way ANOVA results comparing the fracture values of study groups.

\begin{tabular}{|c|c|c|c|c|c|}
\hline Source & $\begin{array}{c}\text { Type III Sum } \\
\text { of Squares }\end{array}$ & df & Mean Square & F & Sig. \\
\hline $\begin{array}{c}\text { Corrected } \\
\text { Model }\end{array}$ & $6.801 \mathrm{E} 6$ & 4 & 1700232.320 & 210.167 & .000 \\
\hline Intercept & $4.401 \mathrm{E} 7$ & 1 & $4.401 \mathrm{E} 7$ & 5439.995 & .000 \\
\hline Groups & 6800929.280 & 4 & 1700232.320 & 210.167 & .000 \\
\hline Error & 364046.100 & 45 & 8089.913 & & \\
\hline Total & $5.117 \mathrm{E} 7$ & 50 & & & \\
\hline $\begin{array}{c}\text { Corrected } \\
\text { Total }\end{array}$ & 7164975.380 & 49 & & & \\
\hline \multicolumn{7}{|c|}{ a. R Squared = .949 (Adjusted R Squared = .945) } \\
\hline
\end{tabular}

TABLE (3) Distribution of fracture patterns for each group.

\begin{tabular}{|c|c|c|}
\hline \multirow{2}{*}{ Group } & \multicolumn{2}{|c|}{ Percentage of failure modes (\%) } \\
\cline { 2 - 3 } & Favorable failure & Unfavorable failure \\
\hline OA & 70 & 30 \\
\hline OH & 40 & 60 \\
\hline OB & 50 & 50 \\
\hline CR & 70 & 30 \\
\hline NR & 80 & 20 \\
\hline
\end{tabular}

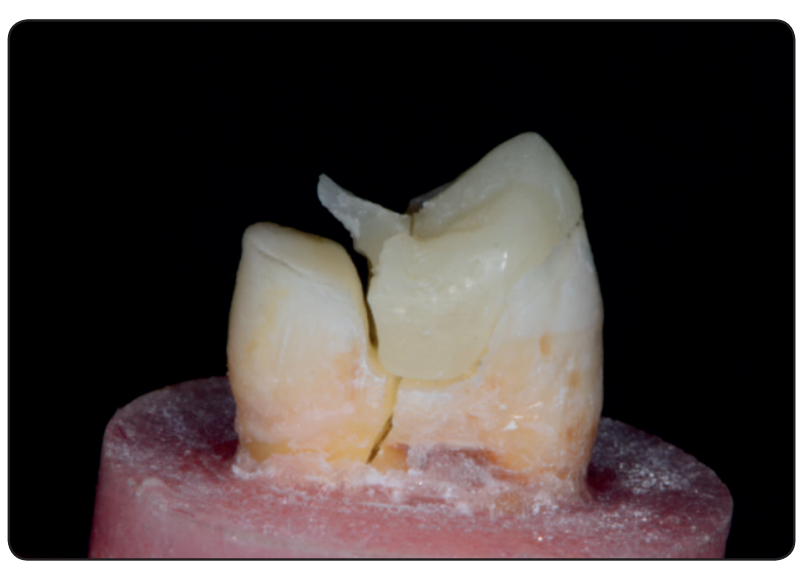

Fig. (1) Photograph representing a non-favorable type of failure with split tooth fracture below the level of bone simulation. 


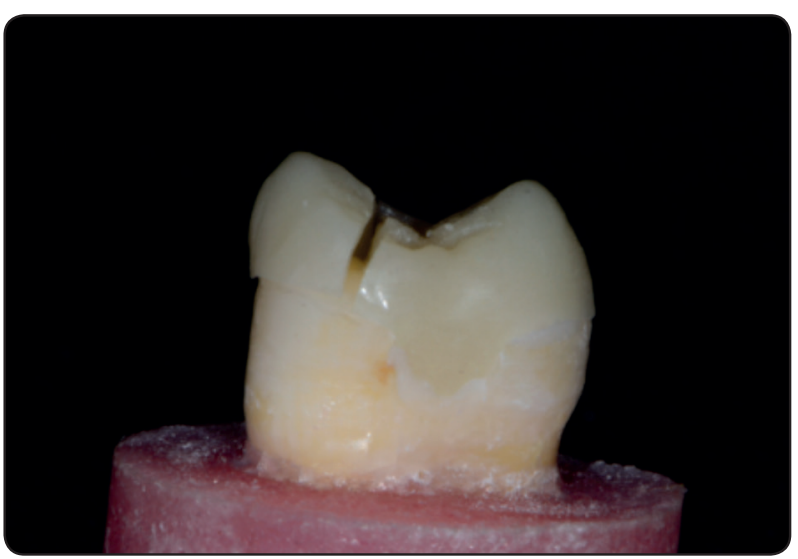

Fig. (2) Photograph representing a favorable type of failure with fracture of the restoration.

\section{DISCUSSION}

Restoration of endodontically treated teeth with application of adhesive protocols offers advantages such as reinforcement of remaining tooth structure, prohibit further loss of tooth structure and offer additional favorable distributions of occlusal loads.,22 The application of adhesive protocols has the capability to reinforce the restored teeth. ${ }^{28,35}$ The employment of bonded cuspal coverage restorations such as onlay and endo-crown restorations to restore endodontically treated teeth with extensive loss of tooth structure constitutes a more conservative strategy in relation to function and esthetics. ${ }^{22,24,35}$ The application of bonded onlay restorations has advantages such as reducing cuspal flexure, cuspal splinting and reinforcement of remaining tooth structure. ${ }^{21,36}$ Following preparation, the decrease of cuspal stiffness has been shown to be substantial, but may be restored with the employment of a bonded restoration. ${ }^{17,37}$ In the present study, the endodontic access cavities was restored with bonded composite resin and definitive ceramic restorations was bonded with resin cement.

Maxillary premolar teeth were utilized in the current investigation because of its susceptibility to cusps deflection and fractures under occlusal stresses. ${ }^{38}$ Tooth structure removal during endodontic procedures and preparation for definitive restoration raises the incidence of cusp deflection and increases the possibility to fracture. The cusp coverage becomes indispensable when the cavity width of the preparation is one half of the buccolingual distance of the tooth or utmost than two thirds of the inter-cuspal distance of the prepared cavity ${ }^{39}$ In the current investigation, the cavity isthmus width was designed to be one-half of the bucco-lingual dimension. Compared with restored teeth, cuspal fracture are infrequently happening in sound teeth due to the buttressing effect of the roof of the pulp chamber and proximal marginal ridges. ${ }^{25,40}$ The endodontic access cavity alone had minimal influence on cuspal stiffness, however the onlay cavity preparation has obvious influence on cuspal stiffness of maxillary premolar teeth and more prone to subsequent fractures. ${ }^{25,41}$

Although previous investigations showed that bonded cusp coverage restorations enhance fracture resistance, ${ }^{1,2,21}$ the literature still uncertain as regard to which type of cusp reduction designs represent the optimum design for management of weakened endodontically treated teeth..$^{21,22}$ Dejak et $\mathrm{a}^{24}$ studied onlays with rounded shoulder, beveled and horizontal preparation designs and revealed that onlay restorations with rounded shoulder designs showed beneficial distribution of occlusal loads. In the current investigation, anatomic, horizontal and beveled cusp reduction designs were employed to acquire the optimum reduction configuration. The data of the current study advocate the presence of variations in the fracture resistance and the failure pattern among cusp coverage restorations of endodontically treated premolar teeth. The reduction parallel to the cusp inclines obviously strengthened the restored teeth. Also, restorable fracture patterns were observed among the anatomic reduction design group. This result is most probably because of the axial orientation of the cusp preparation configuration which would result in an advantageous allocation of occlusal stresses between the restoration and the tooth structure when a compressive load is exerted. Also, that result could be attributed to the enhanced 
resistance to fracture related with beveled design. The bevel designs have been showed to improve the behaviors of restored teeth compared to teeth with non-beveled margin designes. ${ }^{42,43}$ The significance of the cusp inclination and the loading zone $e^{44,45}$ and the position of the residual tooth structure ${ }^{31,34}$ have been described in in previous studies.

The advantage of the onlay preparation designs can be interpreted by the amount of the remaining tooth structure, resulting in favorable distribution of stresses in the tooth and minimized risk of fractures..$^{29,46,47}$ The onlay approach could be considered a suitable method to restore root treated maxillary premolar teeth with the merit of being more conservative than full-coverage restorations ${ }^{48}$ Cusp coverage restorations for endodontically treated teeth represent a conservative approach in terms of function and esthetics. ${ }^{49,50}$ The results of the current study lend support to the possible reasons behind the effectiveness of the onlay restorations. ${ }^{51}$ The average bite force in the premolar teeth rage from $200 \mathrm{~N}$ to about $450 \mathrm{~N}$. Therefore, the tested onlay restorations could be considered strong enough to withstand the clinical bite forces in premolar region.

The null hypothesis that cusp reduction configuration would have no effect on the fracture resistance and fracture mode of onlay restorations of endodontically treated maxillary premolars was rejected. The fracture resistance and failure pattern of onlay restorations were affected by the cusp preparation design.

As limitations to the current study: The findings are applicable only to the utilized ceramic and luting cement and preparation designs evaluated in maxillary premolar teeth. Also, the use of one type of luting cement and restorative material and the use of other types may resulted in different findings. The effect of mechanical cyclic fatigue was not tested. Therefore, more investigations are required to study the influence these variables on the mechanical behavior of cusp coverage onlay restorations.

\section{CONCLUSIONS}

Within the limitations of the present study, the following conclusions were drawn: The root treated maxillary premolars restored with anatomic cusp reduction design displayed greater resistance to fracture and greater rates of restorable fractures than root treated treated maxillary premolars submitted to horizontal and beveled reduction deigns.

\section{REFERENCES}

1. Reeh E, Messer H, Douglas W. Reduction in tooth stiffness as a result of endodontic and restorative procedures. J Endod 1989; 15: 512-516.

2. Rocca G, Krejci I. Crown and post-free adhesive restorations for endodontically treated posterior teeth: from direct composite to endocrowns. Eur J Esthet Dent 2013; 8: 156-179.

3. Randow K, Glantz P O. On cantilever loading of vital and non-vital teeth. An experimental clinical study. Acta Odontol Scand 1986; 44: 271-247.

4. Dietschi D, Duc O, Krejci, I, Sadan A. Biomechanical considerations for the restoration of endodontically treated teeth: A systematic review of the literature, Part II. Evaluation of fatique behavior, interfaces and in vivo studies. Quintessence Int 2008; 39: 117-129.

5. Ng YL, Mann V, Gulabivala K. A prospective study of the factors affecting outcomes of non-surgical root canal treatment: part 1: periapical health. Int Endod J 2011; 44: 583-609.

6. Eliyas S, Jalili J, Martin N. Restoration of the root canal treated tooth. Br Dent J 2015; 218: 53-62.

7. Ichim I, Kuzmnaovic D, Love R. A finite element analysis of ferrule design on restoration resistance and distribution of stress within a root. Int Endod J 2006; 39: 443-452.

8. Heydecke G, Peters MC. The restoration of endodontically treated, single-rooted teeth with cast or direct posts and cores: A systematic review J Prosthet Dent 2002; 87: 380-386.

9. Baratieri L, De Andrada M, Arcari G, Ritter A. Influence of post placement in the fracture resistance of endodontically treated incisors veneered with direct composite resin. J Prosthet Dent 2000; 84: 180-184.

10. Cheung W. A review of the management of endodontically treated teeth: Post, core and the final restoration J Am Dent Assoc 2005; 136: 611-619. 
11. Soares C, Valdivia A, da Silva G, Santana F, Menezes M. Longitudinal clinical evaluation of post systems: a literature review. Braz Dent J 2012; 23: 135-140.

12. Isidor F, Brondum K, Ravnholt G. The influence of post length and crown ferrule length on the resistance to cyclic loading of bovine teeth with prefabricated titanium posts. Int J Prosthodont 1999; 12: 78-82.

13. Stankiewicz N, Wilson P. The ferrule effect: a literature review. Int Endod J 2002; 35: 572-581.

14. Fragou T, Tortopidis D, Kontonasaki E, Evangelinaki E, Ioannidis $\mathrm{K}$, et al. The effect of ferrule on the fracture mode of endodontically treated canines restored with fibre posts and metal-ceramic or all-ceramic crowns. J Dent 2012; 40: 276-285.

15. Dietschi D, Duc O, Krejci, I, Sadan A. Biomechanical considerations for the restoration of endodontically treated teeth: A systematic review of the literature-Part 1. Composition and micro- and macrostructure alterations Quintessence Int 2007; 38: 733-743.

16. Magne P, Knezevic A. Thickness of CAD-CAM composite resin overlays influences fatigue resistance of endodontically treated premolars. Dent Mater 2009; 25 : 1264-1268.

17. Taha N, Palamara J, Messer H. Fracture strength and fracture patterns of root filled teeth restored with direct resin restorations. J Dent 2011; 39: 527-535.

18. Go“hring TN, Peters OA. Restoration of endodontically treated teeth without posts Am J Dent 2003; 16: 313-317.

19. Goracci C, Ferrari M. Current perspectives on post systems: a literature review. Aust Dent J 2011; 56: 77-83.

20. Hannig C, Westphal C, Becker K, Attin T. Fracture resistance of endodontically treated maxillary premolars restored with CAD/CAM ceramic inlays J Prosthet Dent 2005; 94: 342-349.

21. Mondelli R, Ishikiriama S, de Olivera Filho O, Mondelli J. Fracture resistance of weakened teeth restored with condensable resin with and without cusp coverage. J Appl Oral Sci 2009; 17: 161-165.

22. Krifka S, Stang M, Wiesbauer S, Hiller K, Schmalz G, Federlin M. Influence of different cusp coverage methods for the extension of ceramic inlays on marginal integrity and enamel crack formation in vitro. Clin Oral Investig 2009; 13: 333-341.
23. van Dijken JW, Hoglund-Aberg C, Olofsson AL. Fired ceramic inlays: a 6-year follow up. J Dent 1998; 26: 219-25.

24. Magne P, Knezevic A. Thickness of CAD-CAM composite resin overlays influences fatigue resistance of endodontically treated premolars. Dent Mater 2009; 25: 1264-1268.

25. Morimoto S, Vieira G, Agra C, Sesma N, Gil C. Fracture strength of teeth restored with ceramic inlays and overlays. Braz Dent J 2009;143-148.

26. Zadik Y, Sandler V, Bechor R. Analysis of factors related to extraction of endodontically treated teeth. Oral Surg Oral Med Oral Pathol Oral Radiol Endod 2008; 106: 31-35.

27. Fennis W, Kuijs R, Kreulen C, Roeters F, Creugers N, et al. A Survey of cusp fractures in a population of general dental practices. Int J Prosthodont 2002; 15: 559-563.

28. Cotert H, Sen B, Balkan M. In vitro comparison of cuspal fracture resistance of posterior teeth restored with various adhesive restorations. Int J prosthodont 2001; 14: 374-378.

29. Ibrahim A, Richards L, Berekally T. Effect of remaining tooth structure on the fracture resistance of endodontically-treated maxillary premolars: an in vitro study. J Prosthet Dent 2016; 115: 290-295.

30. Lin C, Chang W, Lin YS, Chang Y, Lin YF. Evaluation of the relative contributions of multi-factors in an adhesive MOD restoration using FEA and the Taguchi method. Dent Mater 2009; 25: 1073-1081.

31. Ng C, Dumbrigue H, Al-Bayat M, Griggs J, Wakefield C. Influence of remaining coronal tooth structure location on the fracture resistance of restored endodontically treated anterior teeth. J Prosthet Dent 2006; 95: 290-296.

32. Kalay T, Yildirim T, Ulker M. Effect of different cusp coverage restorations on the fracture resistance of endodontically treated maxillary premolars. J Prosthet Dent 2016; 116: 404-410.

33. Guess P, Schultheis S, Wolkewitz M, Zhang Y, Strub J. Influence of preparation design and ceramic thicknesses on fracture resistance and failure mode of premolar partial coverage restorations. J Prosthet Dent 2013; 110: 264-273.

34. Silva G, Silva N, Soares P, Costa A, Fernandes-Neto A, et al. Influence of different load application devices on fracture resistance of restored premolars. Braz Dent J 2012; 23: 484-489.

35. Nakajima M, Kanno T, Komada W, Miura H, Foxton R, et al. Effect of bonded area and/or fiber post placement on the 
fracture strengths of resin-core reconstructions for pulpless teeth. Am J Dent 2010; 23: 300-304.

36. Brunton P, Cattell P, Burke T, Wilson N. Fracture resistance of teeth restored with onlays of three contemporary toothcolored resin-bonded restorative materials. Prosthet Dent 1999; 82: 167-171.

37. Dias de Souza G, Pereira G, Dias C, Paulillo L. Fracture resistance of premolars with bonded class II amalgams. Oper Dent 2002; 27: 349-353.

38. Shor A, Nicholls J, Phillips K, Libman W. Fatigue load of teeth restored with bonded direct composite and indirect ceramic inlays in MOD class II cavity preparations. Int J Prosthodont 2003; 16: 64-69.

39. Soares P, Santos-Filho P, Martins L, Soares C. Influence of restorative technique on the biomechanical behavior of endodontically treated maxillary premolars. Part I: fracture resistance and fracture mode. J Prosthet Dent 2008; 99: 30-37.

40. Eakle W. Maxwell E, Braly B. Fracture of posterior teeth in adults. J Am Dent Assoc 1986; 112: 215-218.

41. Khera S, Carpenter C, Vetter J, Staley R. Anatomy of cusps of posterior teeth and their fracture potential. Prosthet Dent 1990; 64: 139-147.

42. Dejak B, Mlotkowski A, Romanowicz M. Strength estimation of different designs of ceramic inlays and onlays in molars based on the Tsai-Wu failure criterion. J Prosthet Dent 2007; 98: 89-100.

43. Coelho-de-Souza F, Camacho G, Demarco F, Powers J. Fracture resistance and gap formation of MOD restorations: influence of restorative technique, bevel preparation and water storage. Oper Dent 2008; 33: 37-43.
44. Schmidlin P, Wolleb K, Imfeld T, Gygax M, Lussi A. Influence of beveling and ultrasound application on marginal adaptation of box-only calss II (slot) resin composite restorations. Oper Dent 2007; 32: 291-297.

45. Liu S, Liu I, Xu J, Rong Q, Pan S. Influence of occlusal contact and cusp inclination on the biomechanical character of a maxillary premolar: a finite element analysis . J Prosthet Dent 2014; 112: 1238-1245.

46. Seow L, Toh C, Wilson N. Strain measurements and fracture resistance of endodontically treated premolars testored with all-ceramic restorations. J Dent 2015; 43: 126-132.

47. Edelhoff D, Sorensen JA. Tooth structure removal associated with various preparation designs for posterior teeth. Int J Periodontics Restorative Dent 2002; 22: 241 249 .

48. Mondelli J, Steagall L, Ishikiriama A, de Lima Navarro MF, Soares FB. Fracture strength of human teeth with cavity preparations. J Prosthet Dent 1980; 43: 419-422.

49. Murphy F, Mcdonald A, Petrie A, Palmer G, Stechell D. Coronal tooth structure in root-treated teeth prepared for complete and partial coverage restorations. J Oral Rehabilit 2009; 36: 451-461.

50. Kala T, Yildirim T, Ulker M. Effect of different cusp coverage restorations on fracture resistance of endodontically treated maxillary premolars. Prosthet Dent 2016; 116: 404-410.

51. Craig R, Powers J. Restorative dental materials. $11^{\text {th }}$ ed. Mosby Inc 2002; P68. 